\title{
Effects of Ulinastatin on the Intercellular Adhesion Molecule-1 in Ischemia-Reperfusion Lung of Rat
}

\author{
Duan Ming-ke*, Junbin L, Shengshui X, Wei X, Yizhe G and Muhuo G \\ Department of Thoracic and Cardiovascular Surgery, No2 Hospital Xiamen, Xiamen 361021, Fujian, P.R. China
}

\begin{abstract}
Objective: Ischemia-reperfusion (I/R) injury of the lungs is still a critical problem and a challenge in the field of thoracic and cardiovascular surgery. This study will evaluate effects of Ulinastatin(UTI) on the Intercellular Adhesion Molecule-1(ICAM-1) in the lung ischemia-reperfusion injury of rats. Methods: Single lung in suit warm I/R animal model was used. The rats were subjected to $90 \mathrm{~min}$ left lung ischemia followed by $30 \mathrm{~min}$ and $120 \mathrm{~min}$ of reperfusion separately. The animals were divided into 6 groups. The lungs were removed before ischemia (group pre-I, $n=6$ ), before reperfusion (group pre-R, $n=6$ ), 30 min (group NU-30, $n=6$ ) and 120 min (group NU-120, n=6) after reperfusion began. The UTI (50000 U/kg) was administered just before reperfusion began and the lungs were removed 30 min (group $\mathrm{U}-30, \mathrm{n}=6$ ) and $120 \mathrm{~min}$ (group $\mathrm{U}-120, \mathrm{n}=6$ ) after reperfusion began. For ELISA assay of ICAM-1, the supernatants from left lung tissue homogenates were used.
\end{abstract}

Results: The level of ICAM-1 was increasing as ischemia and reperfusion lasted from Group Pre-R to Group Nu120. When UTI was admitted before reperfusion, the ICAM-1 was down-regulated as seen in the Group U-30 and the group U-120, but in the group U-30 the ICAM-1 was down-regulated significantly $(P<0.05)$.

Conclusion: Ulinastatin protects lung through down-regulate intercellular adhesion molecule-1(ICAM-1) expression in model of experimental ischemia reperfusion injury.

Keywords: Lung; Ischemia-reperfusion injury; Intercellular adhesion molecule-1(ICAM-1); Ulinastatin; Down-regulation

\section{Introduction}

Reperfusion injury after lung transplantation continues to contribute to increased morbidity and mortality. Neutrophils are implicated as mediators of this reperfusion injury in the lung and other organs [1-6]. Numerous investigators have demonstrated improved pulmonary function after ischemia by employing interventions to modulate neutrophil activity. These include the use of leukocytedepleted blood [7], antibodies directed at adhesion molecules expressed on the endothelial surface [8], antibodies directed at the leukocyte integrin CD18 [9] agents aimed at preventing the expression of integrin heterodimers on the leukocyte surface [10], and oxygen free radical scavengers [10]. UTI inhibits release of elastase and cathepsin G from neutrophil granules. We hypothesized that UTI could reduce ischemia reperfusion injury by down regulating the expression of ICAM- 1 as a new of antiadhesion therapy.

\section{Methods}

Pathogen-free adult male SD rats with body weight $250 \mathrm{~g}$ to 350 g (from Animal Laboratory of Shantou University Medical College) were used for all experiments. $36 \mathrm{SD}$ rats were randomly divided into 6 groups. The lungs were removed before ischemia (group pre-I, $n=6$ ), before reperfusion (group pre- $\mathrm{R}, \mathrm{n}=6$ ), 30 (group NU-30, $\mathrm{n}=6$ ) and 120 minutes (group NU-120, $\mathrm{n}=6$ ) after reperfusion began. The UTI (50000 U/kg, gift of Guangdong Tianpu Biochemical Medicine Co. Ltd.) was administered just before reperfusion began and the lungs were removed 30 (group U-30, $\mathrm{n}=6$ ) and 120 minutes (group U-120, $\mathrm{n}=6$ ) after reperfusion.

Animals were initially anesthetized with 30 to $40 \mathrm{mg}$ of intraperitoneal pentobarbital. The animals were then shaved, intubated into the cut of trachea with a 14-gauge catheter, and placed on a small animal ventilator (TKR-200C, made in Jiangxi Teli Anesthesia \& Respiration Instrument Co.) with supplemental oxygen at a rate of 75 cycles per minute, a 1:1.5 of I/E ratio and a maximum of 0.03 to 0.04 MPa peak pressure.
All animals received $0.4 \mathrm{mg}$ of intramuscular atropine after being anesthetized. A left anterolateral thoracotomy was performed via the fifth intercostal space. The left pulmonary hilum was stripped of all neural, vascular, lymphatic, and connective tissue, skeletonizing the left bronchus, pulmonary artery, and pulmonary vein. The inferior pulmonary ligament was divided as it entered the hilum. All dissection was carried out under an operating microscope. Each animal received $50 \mathrm{U}$ of heparin in saline intravenously (total volume, $500 \mu \mathrm{l}$ ) via the dorsal penile vein.

After waiting 5 minutes for circulation of the heparin, the left pulmonary artery, bronchus, and pulmonary vein were sequentially occluded with noncrushing micro vascular clamps. The lungs were kept moist with intermittent application of warm normal saline, and the wounds were covered plastic film to prevent excessive fluid loss. Periods of ischemia were held constant at 90 minutes. At the end of the period of ischemia, the clamps were removed from the vein, bronchus, and artery, and the lungs were allowed to ventilate and reperfuse for periods up to 120 minutes. Animals received $0.5 \mathrm{ml}$ of normal saline via subcutaneous injection for each hour of reperfusion time.

At the end of the period of ischemia, before the clamps were removed from the vein, bronchus, and artery, each animal (group U-30 and group U-120) received $50000 \mathrm{U} / \mathrm{kg}$ of UTI in saline intravenously via the penile vein.

Animals were killed at the end of reperfusion time by clamping the

${ }^{*}$ Corresponding author: Duan Ming-ke, Department of Thoracic Cardiovascular Surgery, No2 Hospital Xiamen, Xiamen 361021, Fujian, P.R. China, Tel: 0086592-6159608; E-mail: mkduans@163.com

Received July 21, 2015; Accepted August 11, 2015; Published August 17, 2015

Citation: Ming-ke D, Junbin L, Shengshui X, Wei X, Yizhe G, et al. (2015) Effects of Ulinastatin on the Intercellular Adhesion Molecule-1 in Ischemia-Reperfusion Lung of Rat. Mod Chem appl 3: 162. doi:10.4172/2329-6798.1000162

Copyright: ( $) 2015$ Ming-ke D, et al. This is an open-access article distributed under the terms of the Creative Commons Attribution License, which permits unrestricted use, distribution, and reproduction in any medium, provided the original author and source are credited. 
right and left superior vena cava and the inferior vena cava. The left atrial appendage was then amputated, and the lungs were flushed with $30 \mathrm{ml}$ of saline by gravity infusion at $30 \mathrm{~cm} \mathrm{H}_{2} \mathrm{O}$ via the right ventricle. The left lung was then removed for analysis as outlined below.

For Enzyme-Linked Immunosorbnent Assay (ELISA) of supernatants from lung tissue homogenates the left rat lungs were used. Samples were immediately snap frozen in liquid nitrogen and stored at $-70^{\circ} \mathrm{C}$. Tissues were homogenized and incubated at $4^{\circ} \mathrm{C}$ in cell lysis buffer

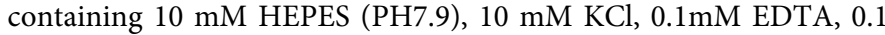
mM EGTA, 1 mM dithiothreitol (DTT), $0.5 \mathrm{mM}$ phenylmethylsulfonyl fluoride (PMSF), and $0.6 \%$ octylphenoxy-polyethoxy- ethanol (Nonidet P-40). Homogenates were then sonicated and centrifuged at $12,000 \mathrm{rpm}$ for $10 \mathrm{~min}$ at $4^{\circ} \mathrm{C}$. Supernatants were assayed in duplicate for total protein; ICAM-1 was measured in supernatant from tissue homogenate by ELISA according to the manufacturer instructions. ICAM-1 was detected with the specific rat immunoassay Quantikine kit from $\mathrm{R} \& \mathrm{D}$ (Minneapolis, MN). The optical density of each well was read at $450 \mathrm{~nm}$ with an NM-600 microplate reader (He Shaoheng Laboratory of Changjiang River Scholars, Shan Tou, China). The protein content was determined by the method of Bradford.

The experimental data were expressed as standard error of the mean, and the $t$ test was used to determine statistical significance $(\mathrm{P}<0.05)$.

\section{Results}

The experimental data were illustrated in Table 1. The level of ICAM-1 was increasing as ischemia and reperfusion lasted from Group Pre-R to Group Nu-120. When UTI was admitted before reperfusion, the ICAM-1 was down-regulated as seen in the Group U-30 and the group U-120, but in the group U-30 the ICAM-1 was down-regulated significantly $(\mathrm{P}<0.05)$.

\section{Discussion}

Primary transplanted graft failure is responsible for high early mortality rates after lung transplantation [11]. It manifests as a mixed vascular and endothelial injury causing increased vascular resistance, pulmonary edema, and impaired gas exchange and represents the sequel of reperfusion injury [12]. Much more studies demonstrated that the neutrophil plays an important role in the etiology of reperfusion injury in all organ systems. During reperfusion activated circulating neutrophils adhere to endothelium. After adhesion neutrophil transmigration occurs followed by release of free radicals and enzymes leading further tissue injury. Consequently, in order to ameliorate neutrophil-related reperfusion injury, some researches have concentrated on leukocyte depletion and blocking neutrophil-endothelial adhesion. Antiadhesion therapy aims to alter the reperfusion process by preventing the interactions between activated neutrophils and endothelium. It can be considered to be specific (the use of monoclonal antibodies against specific adhesion molecules) or nonspecific (the use of soluble substances that saturate the adhesion molecules thereby preventing their interaction with appropriate ligands). ICAM-1 is a ligand for both CD11a/CD18 (LFA-1, lymphocyte function associated antigen-1), and CD11b/CD18 (Mac-1, macrophage-1 antigen) [13,14].

Adhesion molecules on both endothelial cells and neutrophils are key factors that mediate the sequential events of neutrophil rolling, adherence, activation, and emigration in the tissue [14-16]. The contribution of adhesion molecules to the pathogenesis of I/R lung injury has been examined by using Monoclonal Antibodies (MAbs) against specific adhesion molecules: CD11a, CD11b, CD18, ICAM-1, E-selectin, P-selectin, or combination of multiple MAbs [17-19]. These studies revealed that adhesion molecules, espeicially CD11/CD18 and ICAM-1, play a crucial role in the I/R lung injury [17-19]. Because of the interaction between ICAM-1 and its counter-receptor, integrin is believed to be a crucial step in neutrophil adhesion and activation [14-16]. Determining the kinetics of ICAM-1 expression in the lungs may shed some light at least on the reperfusion duration-dependent variation in the neutrophil dependency of lung I/R injury.

Yen-Ta Lu et al. [20] reported that the lung ischemia increased membrane ICAM-1 protein level and that I/R of the lungs caused a biphasic change in ICAM-1 expression with an initial decrease and a late-phase increase. The initial decrease in ICAM-1 protein was prevented by pretreatment with superoxide scavenger SOD and neutrophil depletion, suggesting that superoxide derived from neutrophils (and other leukocytes) plays a great role in the early ICAM1 down-regulation. The late-phase ICAM-1 up-regulation temporally coincided with the I/R-induced elevation of pulmonary micro vascular leakage. Additionally, ICAM-1 MAb prevented I/R-induced increase in pulmonary micro vascular leakage index, suggesting that upregulation of ICAM-1 expression is an important factor contributing to I/R endothelial injury in the lungs. The biphasic change in ICAM1 expression explains the reperfusion duration-dependent variation in the neutrophil dependency of I/R lung injury.

UTI is an acidic glycoprotein with molecular weight 67,000 contained in fresh urine of healthy humans, and it is known as a protease inhibitor of trypsin, chymotrypsin [21]. In addition, UTI has been reported to be effective in the treatment of hemorrhagic shock and septic shock [22,23]. UTI can decrease elastase release from PMNs [24] and suppress the activity of PMN elastase [25]. It can also stabilize lysosomal membranes and suppress the release of lysosomal enzymes [22].

The protective mechanism of UTI to lung reperfusion injury is followed: (1) down-regulating the expression of ICAM-1 induced by TNF- $\alpha$ to prevent the lung injury caused by adhesion [26]; (2) improving the microcirculation and ameliorating the "no reflux" phenomenon; (3) suppressing the activity of PMN elastase to attenuate

\begin{tabular}{|c|c|c|c|c|c|}
\hline & Pre-I & Pre-R & Nu-30 & Nu-120 & U-30 \\
\hline ICAM-1(pg/ml & $908.20 \pm 1$ & $6629.24 \pm 59$ & $9759.54 \pm 77$ & $9827.97 \pm 15$ & $1695.05 \pm 17$ \\
\hline $\begin{array}{c}\text { ICAM-1(ug/mg } \\
\text { protein) }\end{array}$ & $90.98^{\alpha}$ & $.35^{\alpha}$ & $.50^{\alpha \beta}$ & $9.38^{\alpha \beta}$ & $1.00^{\alpha \beta}$ \\
\hline
\end{tabular}

Pre-I: Pre-Ischemia; Pre-R: Pre-Reperfusion; Nu-30: UTI not added before reperfusion, examined at 30min after reperfusion; Nu-120: UTI not added, examined at 120min after reperfusion; U-30: UTI added, examined at 30min after reperfusion; U-120: UTI added, examined at 120min after reperfusion. * $p<0.05$, vs. group Pre-I.

$\# p<0.05$, vs. group Pre-R, group Nu-30, group Nu-120

${ }^{a} p<0.01$, vs. group Pre-I

$\beta p<0.01$, vs. group Pre-R

Table 1: ICAM-1 expression in lung tissue of rats. 
Citation: Ming-ke D, Junbin L, Shengshui X, Wei X, Yizhe G, et al. (2015) Effects of Ulinastatin on the Intercellular Adhesion Molecule-1 in IschemiaReperfusion Lung of Rat. Mod Chem appl 3: 162. doi:10.4172/2329-6798.1000162

the direct and indirect lung injury [27]; (4) inhibiting the activity of anti-coagulation factors including fibrinolysin to prevent the abnormal activity of fibrinogenolysis system [10]; (5) eliminating oxygen free radicals..

Whenever, it is obvious that reperfusion injury is not induced by a simple reaction but occurs in association with various factors. It is suggested that the lung I/R injury is a complicated process. Adhesion molecules on both endothelial cells and neutrophils are key factors that mediate the sequential events of neutrophil rolling, adherence, activation, and emigration in the tissue. Adhesion molecules, especially CD11/CD18 and ICAM-1, play a crucial role in the lung I/R injury. ICAM-1 was one of these factors in our experiment, and the possibility is also suggested that UTI, which is active in down regulating ICAM-1, may control reperfusion injury and improve the result of lung transplantation. The clinical potential of UTI in ameliorating pulmonary reperfusion injury merits further investigation.

\section{Acknowledgement}

This work was funded by Medical Innovative Project of Fujian Province. (No. 2009-CXB-62

\section{References}

1. den Hengst WA, Gielis JF, Lin JY, Van Schil PE, De Windt LJ, et al. (2010) Lung ischemia-reperfusion injury: a molecular and clinical view on a complex pathophysiological process. Am J Physiol Heart Circ Physiol 299: H1283-1299.

2. Li L, Huang L, Vergis AL, Ye H, Bajwa A, et al. (2010) IL-17 produced by neutrophils regulates IFN-gamma-mediated neutrophil migration in mouse kidney ischemia-reperfusion injury. J Clin Invest 120: 331-342.

3. Montalvo-Jave EE, Escalante-Tattersfield T, Ortega-Salgado JA, Piña E, Gelle DA (2008) Factors in the pathophysiology of the liver ischemia-reperfusion injury. J Surg Res 147: 153-159.

4. Feng $\mathrm{Y}$, Zhao H, Xu X, Buys ES, Raher MJ, et al. (2008) Innate immune adaptor MyD88 mediates neutrophil recruitment and myocardial injury after ischemiareperfusion in mice. Am J Physiol Heart Circ Physiol 295: H1311-H1318.

5. Hamada T, Tsuchihashi S, Avanesyan A, Duarte S, Moore C, et al. (2008) Cyclooxygenase-2 deficiency enhances Th2 immune responses and impairs neutrophil recruitment in hepatic ischemia/reperfusion injury. J Immunol 180: 1843-1853.

6. Linfert D, Chowdhry T, Rabb H (2009) Lymphocytes and ischemia-reperfusion injury. Transplant Rev (Orlando) 23: 1-10.

7. Binns OA, DeLima NF, Buchanan SA, Mauney MC, Cope JT, et al. (1996) Neutrophil endopeptidase inhibitor improves pulmonary function during reperfusion after eighteen-hour preservation. J Thorac Cardiovasc Surg 112 607-613

8. Taki-Eldin A, Zhou L, Xie HY, Chen KJ, Yu D, et al. (2012) Triiodothyronine attenuates hepatic ischemia/reperfusion injury in a partial hepatectomy mode through inhibition of proinflammatory cytokines, transcription factors, and adhesion molecules. 178: 646-656.

9. Jaeschke H, Woolbright BL (2012) Current strategies to minimize hepatic ischemia-reperfusion injury by targeting reactive oxygen species. Transplant Rev (Orlando) 26: 103-114.

10. Yang T, Mao YF, Liu SQ, Hou J, Cai ZY, et al. (2010) Protective effects of the free radical scavenger edaravone on acute pancreatitis-associated lung injury. Eur J Pharmacol 630: 152-157.

11. Morrell MR, Patterson GA, Trulock EP, Hachem RR (2009) Acute antibodymediated rejection after lung transplantation. J Heart Lung Transplant 28: 96100

12. Galiè N, Hoeper MM, Humbert M, Torbicki A, Vachiery JL, et al. Guidelines for the diagnosis and treatment of pulmonary hypertension: the Task Force for the
Diagnosis and Treatment of Pulmonary Hypertension of the European Society of Cardiology (ESC) and the European Respiratory Society (ERS), endorsed by the International Society of Heart and Lung Transplantation (ISHLT). Eur Heart J 30: 2493-2537.

13. Springer TA (1990) Adhesion receptors of the immune system. Nature 346: 425-434.

14. Niessen CM, Leckband D, Yap AS (2011) Tissue organization by cadherin adhesion molecules: dynamic molecular and cellular mechanisms of morphogenetic regulation. Physiol Rev 91: 691-731.

15. Zhang J, Alcaide P, Liu L, Sun J, He A, et al. (2011) Regulation of endothelial cell adhesion molecule expression by mast cells, macrophages, and neutrophils. PLoS One 6: e14525.

16. Zarbock A, Ley K (2009) Neutrophil adhesion and activation under flow. Microcirculation 16: 31-42.

17. DeMeester SR, Molinari MA, Shiraishi T, Okabayashi K, Manchester JK, et al. (1996) Attenuation of rat lung isograft reperfusion injury with a combination of anti-ICAM-1 and anti-beta2 integrin monoclonal antibodies. Transplantation 62 1477-1485.

18. Horgan MJ, Ge M, Gu J, Rothlein R, Malik AB (1991) Role of ICAM-1 in neutrophil-mediated lung vascular injury after occlusion and reperfusion. Am J Physiol 261: H1578-1584.

19. Moore TM, Khimenko P, Adkins WK, Miyasaka M, Taylor AE (1995) Adhesion molecules contribute to ischemia and reperfusion-induced injury in the isolated rat lung. J Appl Physiol (1985) 78: 2245-2252.

20. Lu YT, Chen PG, Liu SF (2002) Time course of lung ischemia-reperfusioninduced ICAM-1 expression and its role in ischemia-reperfusion lung injury. $J$ Appl Physiol (1985) 93: 620-628.

21. Umeadi C, Kandeel F, Al-Abdullah IH (2008) Ulinastatin is a novel protease inhibitor and neutral protease activator. Transplant Proc 40: 387-389.

22. Park KH, Lee KH, Kim H, Hwang SO (2010) The anti-inflammatory effects of ulinastatin in trauma patients with hemorrhagic shock. J Korean Med Sci 25 128-134.

23. Okuhama Y, Shiraishi M, Higa T, Tomori H, Taira K, et al. (1999) Protective effects of ulinastatin against ischemia-reperfusion injury. J Surg Res 82: 34-42.

24. Cao ZL, Okazaki Y, Naito K, Ueno T, Natsuaki M, et al. (2000) Ulinastatin attenuates reperfusion injury in the isolated blood-perfused rabbit heart. Ann Thorac Surg 69: 1121-1126.

25. Zaitsu M, Hamasaki Y, Tashiro K, Matsuo M, Ichimaru T, et al. (2000) Ulinastatin, an elastase inhibitor, inhibits the increased mRNA expression of prostaglandin $\mathrm{H} 2$ synthase-type 2 in Kawasaki disease. J Infect Dis 181: 11011109 .

26. Köksoy C, Kuzu MA, Kuzu I, Ergün H, Gürhan I (2001) Role of tumour necrosis factor in lung injury caused by intestinal ischaemia-reperfusion. $\mathrm{Br} \mathrm{J}$ Surg 88 464-468.

27. Carden D, Xiao F, Moak C, Willis BH, Robinson-Jackson S, et al. (1998) Neutrophil elastase promotes lung microvascular injury and proteolysis of endothelial cadherins. Am J Physiol 275: H385-392. 